\title{
Comparative study of hyperon-nucleon interactions of quark model and chiral effective field theory by low-momentum equivalent interactions and $G$ matrices
}

\author{
M. Kohno \\ Physics Division, Kyushu Dental College, Kitakyushu 803-8580, Japan
}

\begin{abstract}
Hyperon-nucleons interactions constructed by two frameworks, the Kyoto-Niigata $\mathrm{SU}_{6}$ quark model and the chiral effective field theory, are compared by investigating equivalent interactions in a low-momentum space and in addition by calculating hyperon single-particle potentials in the lowest-order Brueckner theory in symmetric nuclear matter. Two descriptions are shown to give similar matrix elements in most channels after renormalizing high momentum components. Although the range of the $\Lambda N$ interaction is different in two potentials, the $\Lambda$ single-particle potential in nuclear matter is very similar. The $\Sigma$-nucleus and $\Xi$-nucleus potentials are also found to be similar. These predictions are to be confronted with forthcoming experimental data.
\end{abstract}

PACS numbers: 13.75.Ev, 21.30.Fe, 21.65.-f

\section{INTRODUCTION}

It is basically important to obtain a realistic potential description of baryon-baryon interactions for understanding the properties of baryons and baryonic systems. Baryon-baryon interactions in the strangeness $S=-1$ and $S=-2$ sectors have not been well regulated by experiments, except for a fair amount of data for $\Lambda$ hypernuclei. The construction of these potentials has to rely on an underlying theoretical framework, such as a one boson-exchange potential (OBEP) picture, a constituent quark model, and a chiral effective field theory (EFT). Predictions of these different potential descriptions for hypernuclear phenomenon, for example $\Sigma$ and $\Xi$ hypernuclear bound states, multi hyperon systems, and properties of neutron star matter, naturally vary. Future experimental data will constrain the parameters to allow more solid predictions. Before the experiment, however, it is interesting and important to make a comparison between presently available potential parametrizations to elucidate the character of the underlying theoretical frameworks.

As is known in the nucleon-nucleon $(N N)$ interaction, the direct comparison of the bare potential is not meaningful. We have to consider some effective interactions and quantities closely connected to experimental observables, such as s.p. potentials in the nuclear medium. In this context, equivalent interactions in a low-momentum space [1] have become a useful tool to figure out the properties of baryon-baryon interactions without being obscured by uncertainties in the description of the shortrange part. We call an effective interaction in a restricted space which reproduces the same eigenvalues or $T$-matrices in that space as those of the original full-space interaction an equivalent interaction.

In Ref. [2], we reported the comparison of lowmomentum space equivalent interactions of the Nijmegen OBEP nsc97f [3] and the Kyoto-Niigata $\mathrm{SU}_{6}$ quarkmodel potential fss2 [4] for $\Lambda N$ and $\Sigma N$ interactions, and showed the property of the $\Xi N$ interaction of fss2. For the $\Lambda N$ case, two models provide very similar matrix elements in a low-momentum space, although the shortrange part is considerably different. On the contrary, there is difference in the $\Sigma N$ interaction.

In this paper we extend the study to consider the potential by the chiral EFT [5, 6] and make a comparison with the quark-model potential fss2 in two ways; namely by investigating equivalent interactions in a lowmomentum space and hyperon s.p. potentials in nuclear matter in the framework of the lowest-order Brueckner theory. The elimination of the high-momentum components by considering low-momentum space equivalent interaction enables us to concentrate on features of the $Y N$ interaction relevant to low-energy experimental hypernuclear observables. To consider the implication of baryonbaryon interactions to experimental quantities, it is not sufficient to study the low-momentum interaction. Important correlations inside a low-momentum space and many-body correlations in a high-momentum including the components in a high-momentum space have to be incorporated to obtain physically meaningful quantities. The standard way in nuclear physics is the Brueckner theory. It deals with singular short-range parts of the baryon-baryon interaction, and at the same time incorporates important many-body effects through the Pauli principle and the dispersion effects. Thus we calculate hyperon s.p. potentials in symmetric nuclear matter in the Brueckner theory. The feasible lowest-order calculation accounts for semi-quantitatively the structure dependence of the hyperon-nucleon interactions in the nuclear medium. The s.p. potential is one of most important quantities connected with baryon properties in the nuclear medium, although they are not direct observables. Therefore the hyperon s.p. potentials in the LOBT in symmetric nuclear matter provide a further insight into the properties of the bare hyperon-nucleon interactions.

The fss 2 potential is the most recent model by the Kyoto-Niigata group [4, 7], in which an effective gluonic interaction and long-ranged one-boson exchanges between quarks are considered in the resonating group method (RGM) for two constituent-quark clusters. This 
fss2 [4] achieves comparable accuracy in the $N N$ sector to modern realistic $N N$ potentials. The extension of the potential to the strangeness $S=-1$ and $S=-2$ sectors on the basis of the parameters fixed in the $N N$ sector has been shown [4] to be less ambiguous than the OBEP parametrization. In fact, the prediction of the overall repulsive nature of the $\Sigma$-nucleus potential before experiments is supported by analyses $[8,9,10]$ of the $\left(\pi^{-}, K^{+}\right)$ $\Sigma$ production inclusive spectra [8, 11]. The microscopic calculation of the $\Sigma$-nucleus s.p. potential in finite nuclei 12] further demonstrated that the fss 2 potential actually reproduces the subtle structure of the weak surface attraction and the repulsion inside a nucleus which is indicated by the analyses [13] of the shift and width of $\Sigma^{-}$ atomic levels.

The chiral EFT potentials in the strangeness $S=-1$ and $S=-2$ sector have been recently developed by the Jülich group [5, 6], as the extension of the nucleonnucleon case [14]. This description uses pseudoscalarmeson exchanges and flavor $\mathrm{SU}_{3}$ invariant contact terms, regularized by a cut-off mass of around $600 \mathrm{MeV}$. At present the interaction is derived in the leading order. Parameters of the contact terms, 5 in number in the $S=-1$ sector and an additional one parameter in the $S=-2$ sector, are determined by fitting to available experimental data. Because the description for the shortrange part is considerably different from that of fss 2 , it is worthwhile to compare two potentials.

In Sec. II, we briefly describe the basics of the equivalent interaction theory in a model space. Results of numerical calculations in ${ }^{1} S_{0}$ and ${ }^{3} S_{1}$ channels are presented in Sec. III for $\Lambda N, \Sigma N$, and $\Xi N$ interactions. We also present the $\Lambda, \Sigma$, and $\Xi$ s.p. potentials in symmetric nuclear matter at various Fermi momenta between $k_{F}=0.75$ and $1.45 \mathrm{fm}^{-1}$. Section IV summarizes the results of the present paper.

\section{EQUIVALENT INTERACTION}

Suzuki and Lee [15, 16] proposed in 1980 the basic idea to construct the energy-independent hermitian equivalent Hamiltonian in a model space $P$. Their consideration is closely related to the recent development of low-momentum interactions [1]. It is elementary to observe that the eigenvalues of the original Hamiltonian $H$ do not change when $H$ is transformed by a similarity transformation, namely by a regular matrix $X$ and its inverse $X^{-1}$ as $H \Rightarrow H^{\prime} \equiv X^{-1} H X$. It is easy to see that if a decoupling condition $Q X^{-1} H X P=0$ holds with $Q=1-P, P X^{-1} H X P$ becomes the equivalent Hamiltonian $H_{\text {eff }}$ in the model space $P$. Thus the task to find $H_{\text {eff }}$ is reduced to determine $X$ which satisfies $Q X^{-1} H X P=0$. It is sufficient first to consider a regular matrix $X$ in the following form.

$$
X=\left(\begin{array}{c}
1,0 \\
\omega, 1
\end{array}\right), \quad \text { then } \quad X^{-1}=\left(\begin{array}{c}
1,0 \\
-\omega, 1
\end{array}\right)
$$

The mapping matrix $\omega=Q \omega P$, which connects the $P$ and $Q$ spaces, plays a central role in the construction of $H_{\text {eff }}$. The decoupling condition $Q X^{-1} H X P=0$ now reads:

$$
Q H P+Q H Q \omega-\omega P H P-\omega P H Q \omega=0 .
$$

Because this is a non-linear equation for $\omega$, we have to use some iteration method to solve it. Determining the mapping operator $\omega$, we obtain an energy-independent equivalent Hamiltonian in the model space $P$ as $H_{\text {eff }}=$ $P H P+P H Q \omega P$. This equivalent Hamiltonian is not hermitian at this stage. If we utilize a unitary matrix $\tilde{X}$ in the following Okubo form [17] constructed from $\omega$ of Eq. (1) to transform the original $H$, we obtain the hermitian Hamiltonian.

$$
\tilde{X}=\left(\begin{array}{c}
1,-\omega^{\dagger} \\
\omega, 1
\end{array}\right)\left(\begin{array}{c}
1+\omega^{\dagger} \omega, 0 \\
0,1+\omega \omega^{\dagger}
\end{array}\right)^{-1 / 2}
$$

Subtracting the kinetic part, we can define an equivalent interaction in the model space. In the case of the equivalent interaction in a two-body problem, for example the elimination of high-momentum components, the procedure is transparent, because many-body correlations do not appear.

The actual calculation of the mapping operator $\omega$ is carried out by the method-2 in Ref. [18]. The extension to the hyperon-nucleon case, in which several baryonchannels couple each other and there appears an antisymmetric spin-orbit coupling absent in the $N N$ interaction, is straightforward. However, we encounter numerical troubles in some cases, i.e. in the $T=\frac{1}{2} \Sigma N{ }^{1} S_{0}$ and ${ }^{3} S_{1}$ channels and the $T=0 \Xi N{ }^{1} S_{0}$ channel, when the threshold of another baryon channel is located in the low-momentum space. The extended method-2 yields oscillatory behavior of the matrix elements of the equivalent interaction as a function of the momentum that varies as mesh points are altered. One tentative remedy is to use rather coarse mesh points to obtain smooth $k$ dependence. But, this does not always work. It requires in future a new numerical method or a more radical reformulation such as introducing a channel-dependent cutoff [19] to resolve the problem. Because the aim of the present evaluation is to compare characters of different baryon-baryon interactions and not to do exact structure calculations on the basis of the low-momentum equivalent interaction, we present the results with the oscillatory behavior in case it appears.

$G$-matrix calculations for hyperons in symmetric nuclear matter are carried out, using the continuous prescription for intermediate spectra. Hyperon s.p. potentials are determined self-consistently. Details are reported in Ref. [20]. In calculating the hyperon-nucleon $G$ matrices for chiral EFT, we use the nucleon s.p. potential obtained by fss 2 to focus on the properties of the hyperon-nucleon interactions. 


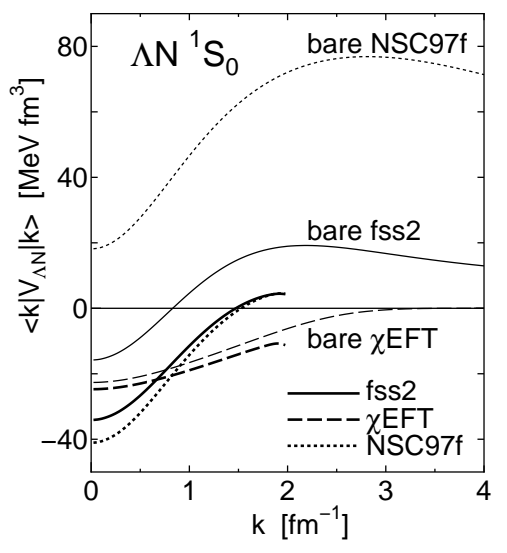

FIG. 1: Diagonal matrix elements of the equivalent interaction in the low-momentum space with $\Lambda=2 \mathrm{fm}^{-1}$ for the $\Lambda N{ }^{1} S_{0}$ channel, using the quark-model potential fss2 [4], the Nijmegen potential NSC97 [3] , and the chiral EFT potential $(\chi \mathrm{EFT})$ [5] with a cut-off mass of $600 \mathrm{MeV}$. Bare matrix elements are shown by thin curves.

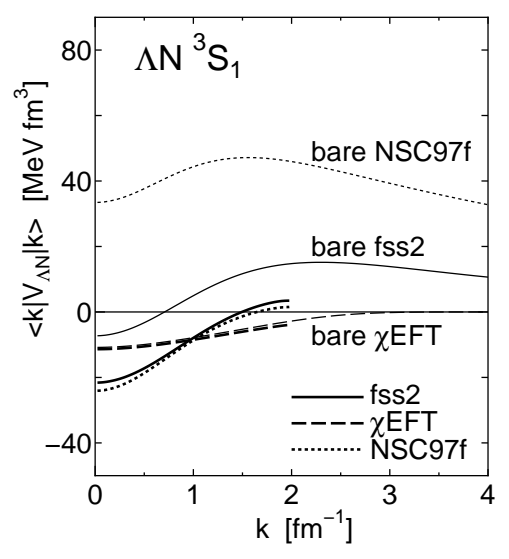

FIG. 2: Same as Fig. 1, but for the $\Lambda N^{3} S_{1}$ channel.

\section{CALCULATED RESULTS}

We calculate equivalent $\Lambda N, \Sigma N$ and $\Xi N$ matrix elements in the low-momentum space with the cut-off value of $\Lambda=2.0 \mathrm{fm}^{-1}$ for the ${ }^{1} S_{0}$ and ${ }^{3} S_{1}$ partial waves, starting from the Kyoto-Niigata $\mathrm{SU}_{6}$ quark-model potential fss2 [4] and the chiral EFT potential [5, 6]. This momentum scale should be regarded as a representative one for which the potential dependence of the description of high momentum components has been shown [1] to disappear in the case of the $N N$ interaction. As explained in Ref. 2], we use the energy-independent version of the quark-model potential [21] that eliminates the energy dependence originating the RGM treatment of the quark clusters. Note that the short-range part of the baryonbaryon interaction in the quark model is constructed by a RGM framework for nonrelativistic quark-clusters, while that of the chiral EFT potential is influenced by the contact terms determined by phenomenological fitting.

\section{A. $\Lambda N$ interaction}

Figures 1 and 2 show the low-momentum space diagonal matrix elements of the equivalent $\Lambda N$ interaction in the ${ }^{1} S_{0}$ and ${ }^{3} S_{1}$ channels, respectively, together with bare matrix elements. In this case we include the equivalent interaction of the Nijmegen potential NSC97f [3] , in addition to the quark model potential fss2 [4] and the chiral EFT potential [5] with a cut-off mass of $600 \mathrm{MeV}$.

As reported already in Ref. [2], the NSC97f and the fss 2 provide very similar matrix elements in the lowmomentum space, in spite of the large difference in the short-range part as the bare matrix elements indicate. On the other hand, the $k$-dependence of the chiral EFT potential differs from other two potential, though the overall attractive strength is of the same order. Because of the regularization with the cutoff mass of $600 \mathrm{MeV}$, the high-momentum component of the chiral EFT potential is small and the equivalent interaction is not so much different from the bare interaction in the low-momentum space. The weak $k$-dependence suggests that the chiral EFT interaction is more short-ranged than other two potentials in both ${ }^{1} S_{0}$ and ${ }^{3} S_{1}$ channels. Note that in the $\Lambda N$ case, a direct isovector $\pi$ exchange process is absent. In the ${ }^{3} S_{1}$ channel, a considerable amount of the attractive contribution is expected from the $\Lambda N-\Sigma N$ coupling through the $\pi$ exchange. In the cases of fss 2 and NSC97f, the attraction in a low-momentum space comes from this coupling in a high-momentum space with the tensor component of the $\pi$ exchange. In contrast, the small difference between bare and low-momentum space matrix elements in the case of chiral EFT implies that the coupling effect in a high-momentum space is incorporated in the parameter of the contact terms.

Diagonal matrix elements of the effective interaction in momentum space determine baryon s.p. potentials in nuclear matter. Because properties of the s.p. potential can be more directly inferred from experimental data, it is useful to present the calculated $\Lambda$ s.p. potential from the $\Lambda N$ interaction. We can consider the Hartree potential obtained by the equivalent interaction in the low-momentum space. However, we prefer to use the standard lowest-order Brueckner theory, in which some important many-body effects are incorporated. The calculated $\Lambda$ s.p. potential from the two bare potentials, fss2 and chiral EFT, are shown in Fig. 3. The real part is very similar in its magnitude and $k_{F}$ dependence. It is not easy to detect the difference of the $k$ dependence observed in the equivalent interactions in the ${ }^{1} S_{0}$ and ${ }^{3} S_{1}$ channels. The imaginary part of the s.p. potential indicates the strength of the $\Lambda N-\Sigma N$ coupling. At low momentum, the chiral EFT potential gives slightly larger imaginary strength. The weaker imaginary potential from the chiral EFT than that from fss2 at large $k$ region is due to the weak $\Lambda N-\Sigma N$ coupling inherent in the cutoff mass of $600 \mathrm{MeV}$. As noted above, the coupling effect at high-momentum region may be renormalized into the parameter of the contact terms in the chiral EFT 


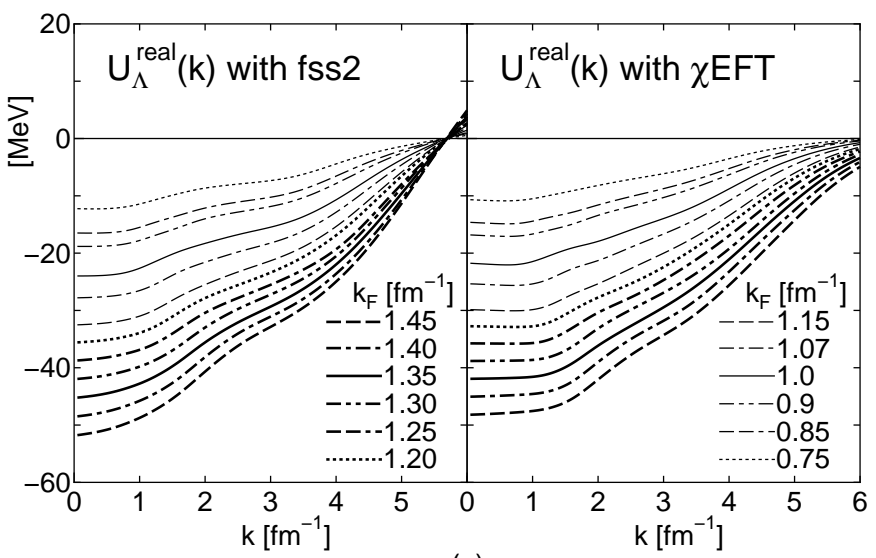

(a)

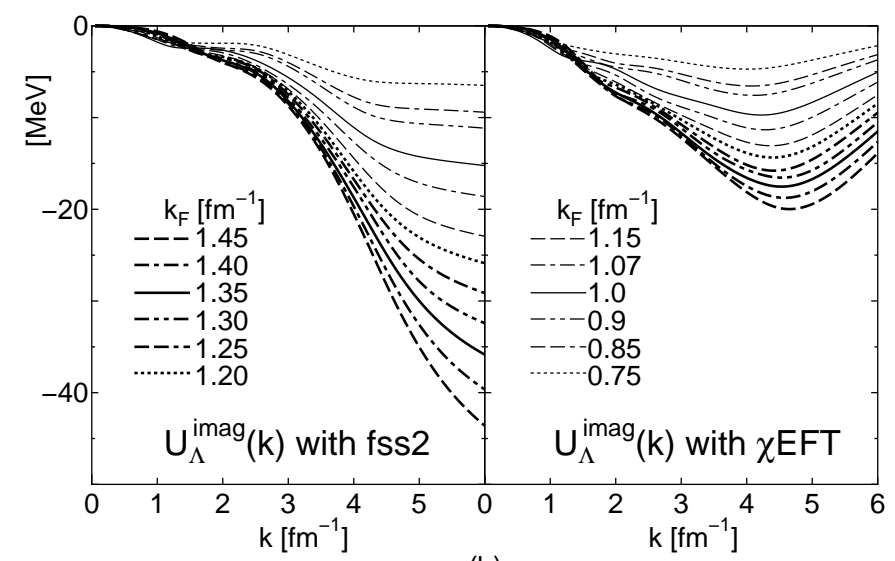

(b)

FIG. 3: Momentum dependence of $\Lambda$ s.p. potential in symmetric nuclear matter at various Fermi momenta $k_{F}$ : (a) real part and (b) imaginary part. The calculations are in the lowest-order Brueckner theory with the continuous prescription for the intermediate spectra. The left panel shows the result of the quark-model potential fss2 [4], and the right panel those of the potential of the chiral EFT ( $\chi \mathrm{EFT})$ [5] with a cut-off mass of $600 \mathrm{MeV}$.

potential and thus the explicit $\Lambda N-\Sigma N$ coupling at the large momentum region is weak in this parametrization.

As a whole, three bare potentials, fss2, NSC97f, and chiral EFT, for the $\Lambda N$ interaction provide similar description of the $\Lambda$ s.p. potential. In literature [22, 23, 24] we find that the energy of the hypertriton is well reproduced by three potentials: namely $E_{3} H=-2.30$, -2.487 , and $-2.34 \mathrm{MeV}$ for NSC97f, fss2, and chiral EFT, respectively, compared with the empirical value of $-2.354 \pm 0.050 \mathrm{MeV}$. However, the difference observed in Figs. 1 and 2 for the $k$ dependence of the equivalent interaction is probably detectable in some experimental observables.

Finally, it is worth to comment on an unresolved problem of the microscopic understanding of the small $\Lambda$ s.p. spin-orbit potential. Experimentally, it has been established 25] that the spin-orbit splitting of the $\Lambda$ s.p. levels in nuclei is very small. It is helpful to consider the Scheerbaum factor $S_{\Lambda}$ [26] calculated in nuclear matter to relate the strength of the $\Lambda$-nucleus spin-orbit potential to the two-body $\Lambda N$ interaction. The $\Lambda$-nucleus s.p. potential is well simulated by

$$
U_{\Lambda}^{\ell s}(r)=-\frac{\pi}{2} S_{\Lambda} \frac{1}{r} \frac{d \rho(r)}{d r} \ell \cdot \sigma
$$

where $\rho(r)$ is a nucleon density distribution. The necessary value of $S_{\Lambda}$ to explain experimental data is about $-3.2 \mathrm{MeV} \cdot \mathrm{fm}^{5}$. In contrast, three potentials considered here gives $S_{\Lambda}=-15.4,-12.2$ and $+4.8 \mathrm{MeV} \cdot \mathrm{fm}^{5}$ for NSC97f, fss2, and chiral EFT, respectively, in normal symmetric nuclear matter, namely $k_{F}=1.35 \mathrm{fm}^{-1}$. The quark model suggests that antisymmetric spin-orbit component of the two-body spin-orbit interaction may be important to cancel the ordinary spin-orbit interaction. However, this mechanism does not work quantitatively in fss2. Other two potentials do not contain the antisymmetric spin-orbit component. It is significant that the chiral EFT potential predicts an opposite sign for the $\Lambda$ s.p. spin-orbit potential in the present leading order construction.

\section{B. $\Sigma N$ interaction}

Figure 4 shows the low-momentum space diagonal matrix elements of the equivalent $\Sigma N$ interaction in the ${ }^{1} S_{0}$ and ${ }^{3} S_{1}$ channels for the isospin $T=\frac{1}{2}$ and $\frac{3}{2}$, respectively, together with bare matrix elements for the quarkmodel potential fss2 [4] and the chiral EFT potential with the cutoff mass of $600 \mathrm{MeV}$.

It is notable that the equivalent interactions of the quark model fss2 and the chiral EFT potential are very similar except for the ${ }^{3} S_{1} T=\frac{1}{2}$ channel. It is known that the $\Sigma N^{1} S_{0} T=\frac{3}{2}$ state consists of the same $(2,2)$ flavor $\mathrm{SU}_{3}$ symmetric component of the Elliott notation $(\lambda, \mu)$ as the $N{ }^{1} S_{0}$ state. Thus, this channel is expected to hold a rather strong attraction. This character is manifested in the $J^{\pi}=0^{+}{ }_{\Sigma}^{4} \mathrm{He}$ bound state seen in the ${ }^{4} \mathrm{He}\left(K^{-}, \pi^{-}\right)$reactions [27, 28]. The chiral EFT potential also also has this attraction, although the $k$ dependence is gentle as in the $\Lambda N$ equivalent interactions.

The quark model picture has been known from the earlier studies [7, 29] to give a definite prediction that the $\Sigma N{ }^{3} S_{1} T=3 / 2$ state should be strongly repulsive due to the quark Pauli effect, which has no explicit counterpart in the OBEP parametrization. The repulsive character persists in the low-momentum space. Owing to the spin and isospin weight factors, this ${ }^{3} S_{1} T=3 / 2$ state dominantly contributes to the $\Sigma$ s.p. potential in the nuclear medium, as will be explicitly shown below in the calculated $\Sigma$ s.p. potential. Analyses [8, 9, 10] of the $\left(\pi^{-}, K^{+}\right)$ $\Sigma$ formation inclusive spectra [8] have supported the overall repulsive nature of the $\Sigma$-nucleus potential. Note that the actual calculation [12] in finite nuclei shows that the 


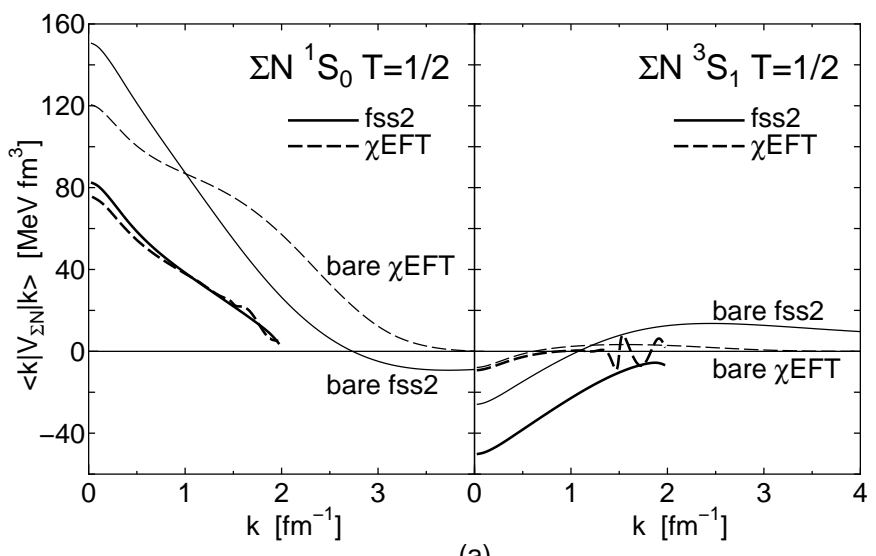

(a)

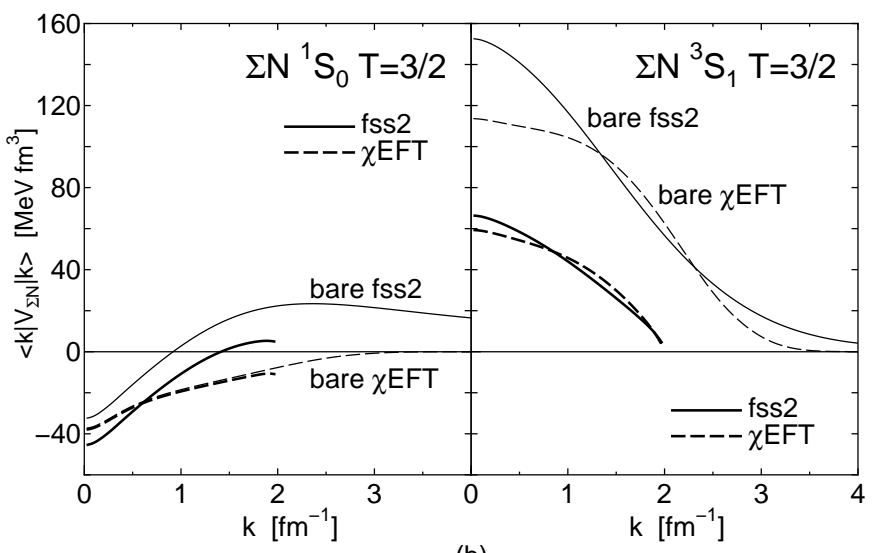

(b)

FIG. 4: Diagonal matrix elements of the equivalent interaction in the low-momentum space with $\Lambda=2 \mathrm{fm}^{-1}$ for the $\Sigma N{ }^{1} S_{0}$ and $\Sigma N{ }^{3} S_{1}$ channels, using fss2 [4] and chiral EFT ( $\chi$ EFT) [5]: (a) isospin $T=\frac{1}{2}$ and (b) $T=\frac{3}{2}$.

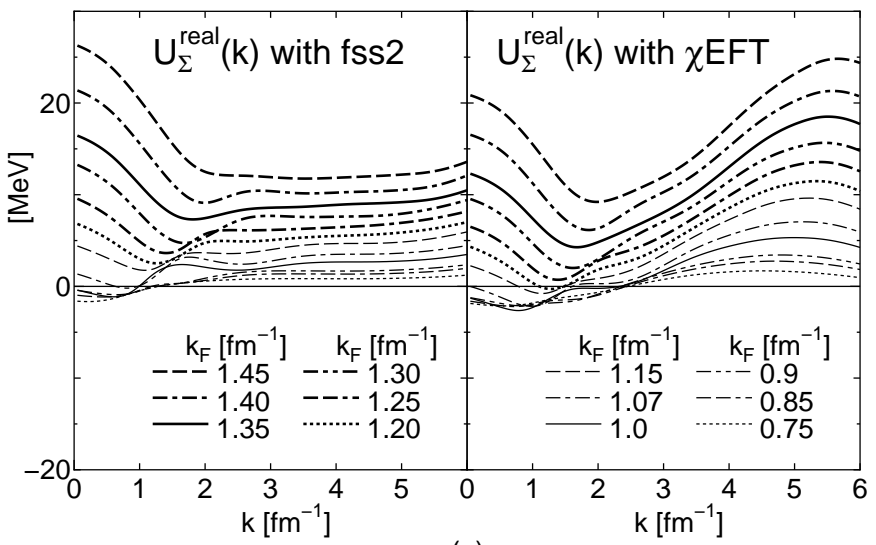

(a)

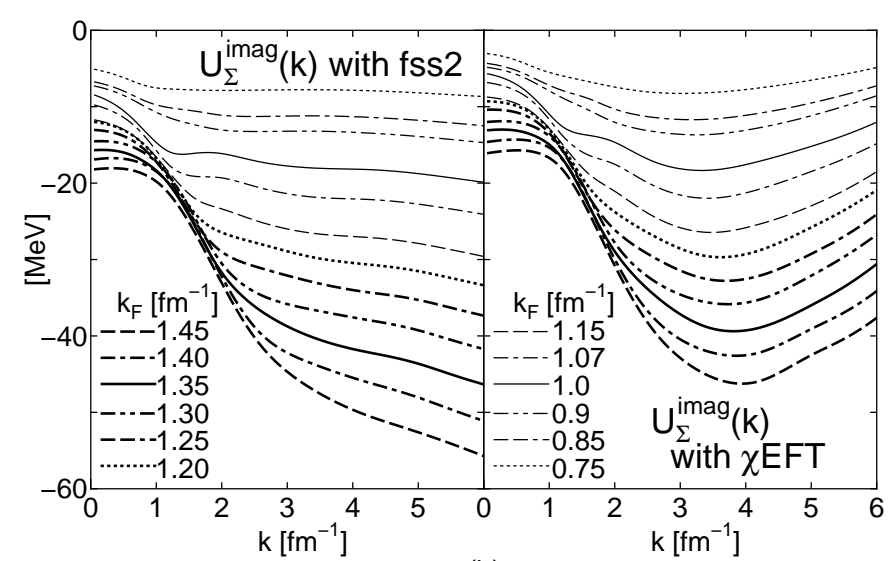

(b)

FIG. 5: Same as Fig. 3, but for the $\Sigma$ s.p. potential.

we obtain weak attractive potential at the surface region of nuclei that is necessary to account for the energy shift of $\Sigma^{-}$atomic levels.

It is interesting to see that the matrix elements in the ${ }^{3} S_{1} T=\frac{3}{2}$ channel are similar for the fss 2 and the chiral EFT. While the repulsive character is dictated by the quark Pauli effect in the fss2, the parameter of the contact term determined phenomenologically is responsible for this repulsion in chiral EFT.

Calculated $\Sigma$ s.p. potentials in symmetric nuclear matter are shown in Fig. 5. Two potentials predict very similar patterns for the real part both in the $k$ dependence and in the $k_{F}$ dependence. The size of the imaginary strength is also seen to be resembling except for the region beyond $k \sim 4 \mathrm{fm}^{-1}$.

\section{C. $\Xi N$ interaction}

Figure 4 shows the low-momentum space equivalent $\Xi N$ interaction in the ${ }^{1} S_{0}$ and ${ }^{3} S_{1}$ channels for the isospin $T=\frac{1}{2}$ and $\frac{3}{2}$, respectively, together with the bare matrix elements up to $k=4 \mathrm{fm}^{-1}$. In the $T=1$ channel, two potentials have similar repulsive characters both in the bare and equivalent interactions.

The quark-model potential provides fair attraction in the $T=0^{1} S_{0}$ channel. The most part of this attraction comes from the $\Xi N-\Lambda \Lambda-\Sigma \Sigma$ coupling. This can be checked by observing that if we switch off the baryonchannel coupling, the matrix elements are close to those of the bare interaction. In such a situation, it is important to consider the effect of the baryon-channel coupling in the $P$ space to obtain more physically meaningful information. The situation is the same in chiral EFT, though the resulting attraction is very small in magnitude. Note that in the chiral EFT theory an additional parameter has to be introduced in the ${ }^{1} S_{0}$ channel when 


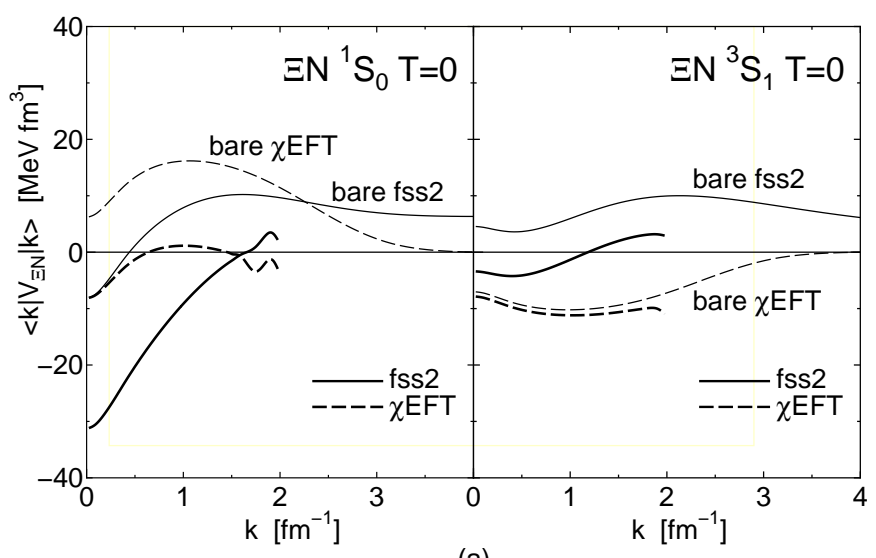

(a)

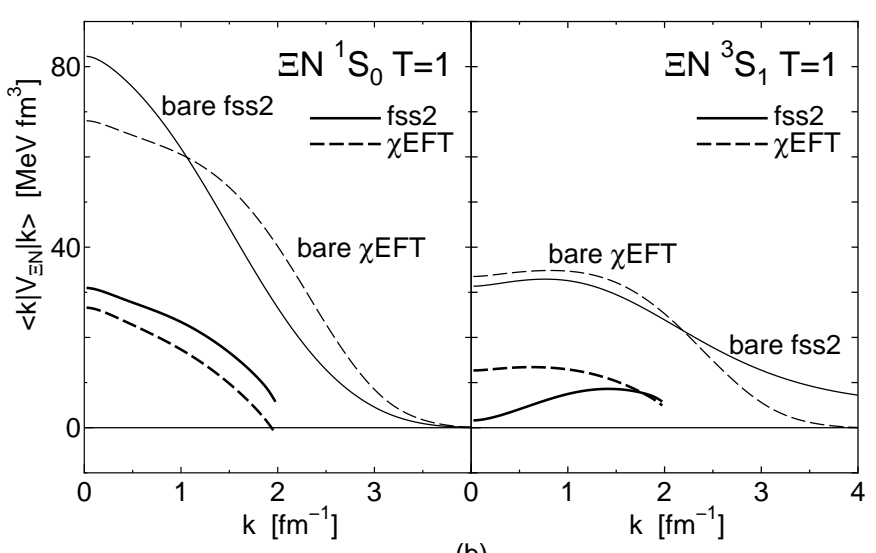

(b)

FIG. 6: Same as Fig. 4, but for the $\Xi N{ }^{1} S_{0}$ and $\Xi N{ }^{3} S_{1}$ channels: (a) isospin $T=0$ and (b) isospin $T=1$. The chiral EFT $(\chi \mathrm{EFT})$ potential is from [6].

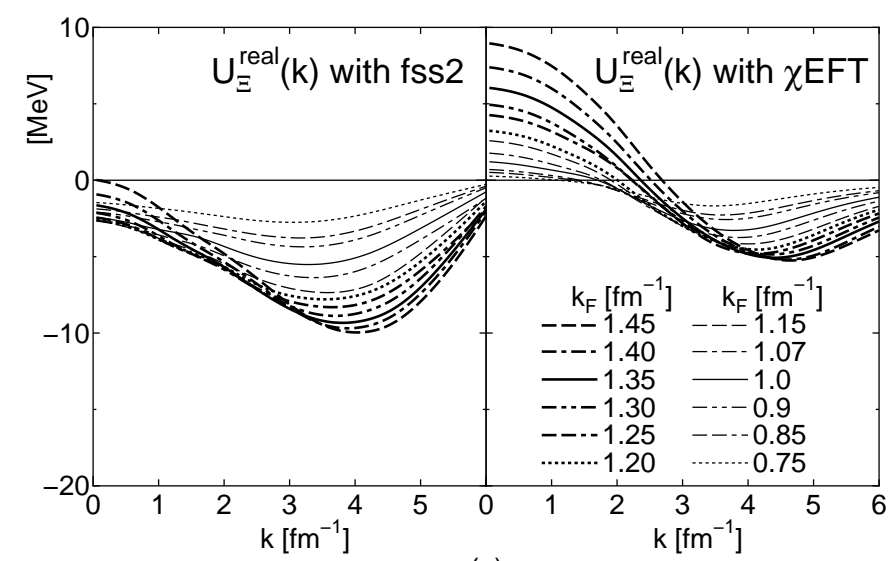

(a)

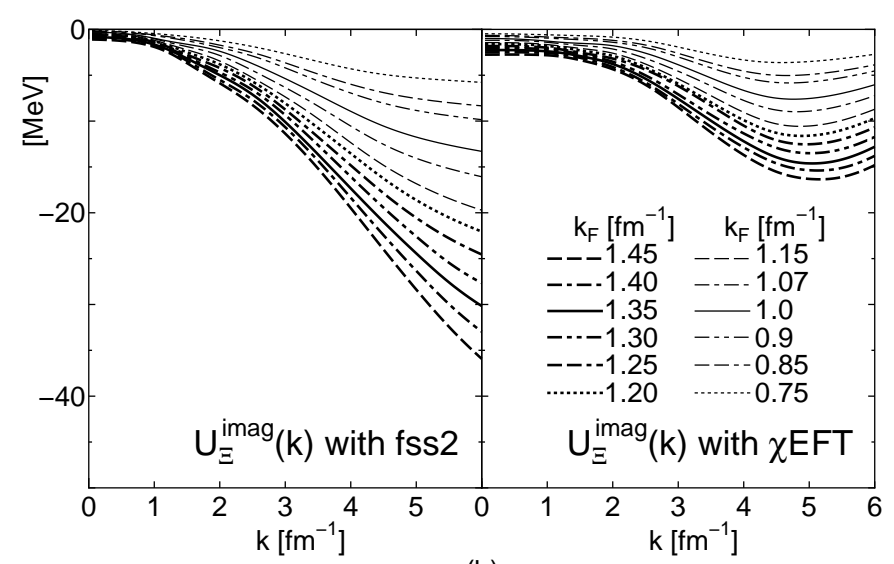

(b)

FIG. 7: Same as Fig. 3, but for the $\Xi$ s.p. potential. The chiral EFT ( $\chi \mathrm{EFT})$ potential is from [6].

extending to the $S=-2$ sector from the $S=-1$ sector.

The ${ }^{3} S_{1} T=0$ state is classified to the pure $(11)_{a}$ state in the flavor $\mathrm{SU}_{3}$ symmetry and no baryon-channel coupling appears in this state. The quark model [4] predicts that the bare $\Xi N$ interaction is already weak. Figure 11 shows that the low-momentum equivalent $\Xi N$ interaction in this partial wave becomes slightly attractive.

The quark-model potential fss2 [4] predicts that the $\Xi N$ interactions in ${ }^{3} S_{1}$ channels are weak. For the estimation of the $\Xi$-nucleus s.p. potential in nuclear medium, we expect an attractive contribution from the ${ }^{1} S_{0} T=0$ state but a repulsive contribution from the ${ }^{1} S_{0} T=1$ state. Higher partial waves can influence the sign of the $\Xi$-nucleus s.p. potential, although it is unlikely that they bring about sizable net attractive or repulsive contributions. The calculated $\Xi$ s.p. potentials in symmetric nuclear matter in the LOBT are shown in Fig. 7. The potential from the fss2 is seen to be weak. The tendency that the attractive strength is largest at $k=3 \sim 4$ is owing to the net contribution of $\Xi N p$ waves.
Although the potential is attractive in infinite matter, the calculation in finite nuclei [12] shows that the $\Xi$ s.p. potential is weakly attractive at the nuclear surface, and oscillates around zero inside the nucleus. Judging from Fig. 7, a more repulsive $\Xi$ s.p. potential in finite nuclei is expected from chiral EFT. The presently available $\left(K^{-}, K^{+}\right)$spectrum [30] at the $\Xi^{-}$production threshold region is shown in Ref. [31] to be consistent with the weakly repulsive $\Xi$ potential. The prediction is to be confronted with an experimental data with better accuracy obtained soon from J-PARC [32].

\section{SUMMARY}

We have compared two descriptions of the hyperonnucleon interactions, the Kyoto-Niigata quark-model potential fss2 [4] and the chiral EFT potential [5, 6], by calculating low-momentum space equivalent interactions and hyperon s.p. potentials in the LOBT in symmet- 
ric nuclear matter obtained from these bare potentials. The purpose is to elucidate the similarity and the difference in the $\Lambda N, \Sigma N$ and $\Xi N$ interactions between the quark model and the chiral EFT theory. The former model is based on a resonating-group method for two constituent-quark clusters with an effective gluonic interaction and long-ranged one-boson exchanges between quarks. The energy dependence inherent in the RGM treatment is eliminated by the method in Ref. [21]. The latter parametrization uses pseudoscalar-meson exchanges and flavor $\mathrm{SU}_{3}$ invariant contact terms, regularized by a cut-off mass of around $600 \mathrm{MeV}$. Parameters of the contact terms, 5 in the $S=-1$ sector and an additional one parameter in the $S=-2$ sector, are determined by fitting to available experimental data. Because of the difference in the description for the short-range part, it is worthwhile to compare two potentials.

In the previous paper [2], we showed that the $\Lambda N$ equivalent interaction in the low-momentum space is almost identical for the quark model fss 2 and the Nijmegen OBEP model NSC97f [3]. In this paper, we have found that the leading order chiral EFT interaction gives matrix elements of the equivalent interaction which have different $k$ dependence from the fss 2 . This difference is not visible in the $\Lambda$ s.p. potential in the nuclear medium, although it is probably detectable in some observables in future experiments. Note that there is an unresolved problem of describing very small spin-orbit splitting of the $\Lambda$ hyperon in nuclei. $G$-matrix calculations, in which effects of the $\Lambda N-\Sigma N$ coupling in the nuclear medium are taken care of, show that fss 2 does not provide a small $\Lambda$ nucleus spin-orbit potential necessary to account for the empirical data, in spite of the tendency of the cancellation of the ordinary and antisymmetric spin-orbit components. On the other hand, the chiral EFT potential having no antisymmetric spin-orbit component predicts a small but opposite sign for the spin-orbit potential. It is interesting if the effects of the next leading prder corrections is revealed in the future analysis.

As for the $\Sigma N$ interactions, the quark model fss 2 and the chiral EFT potential mostly give similar matrix elements of the equivalent interactions in a low-momentum space, except for the ${ }^{3} S_{1} T=\frac{1}{2}$ state. It is interesting to see that the repulsion in the ${ }^{3} S_{1} T=\frac{3}{2}$ state predicted by the quark model because of the quark Pauli effect is reproduced as well in the chiral EFT parametrization. This similarity reflects in that calculated $\Sigma$ s.p. potentials in symmetric nuclear matter also resemble in their magnitude, momentum dependence, and $k_{F}$ dependence. The prediction of the repulsive potential for the $\Sigma$ hyperon embedded in the nuclear medium is not common among several baryon-baryon interaction parametrizations. Therefore checks by forthcoming experiments, for example in the J-PARC project [32], will be very important for understanding the $\Sigma N$ interaction.

The resemblance of the two interactions holds also in the $\Xi N$ interaction. The fss 2 interaction provides weakly attractive s.p. potentials in symmetric nuclear matter. The chiral EFT interaction tends to give slightly more repulsive s.p. potentials because of the lack of attraction in the ${ }^{1} S_{0} T=0$ channel. The microscopic calculation [12] of the $\Xi$-nucleus potential in finite nuclei shows that the fss 2 predicts an almost zero potential. At the surface region, the potential is weakly attractive and inside the nucleus the potential fluctuate around zero. Such weak $\Xi$-nucleus potential is shown in Ref. [31] to be able to account for the existing $\left(K^{-}, K^{+}\right)$spectrum at the threshold region 30]. This experimental data is based on the small number of counts and thus may not be accurate enough to conclude the strength of the $\Xi$-nucleus potential. We expect new $\Xi$-production spectrum data with better accuracy from the J-PARC experiments [32], which will provide important information on the baryon-baryon interaction in the $S=-2$ sector.

\section{Acknowledgments}

The author is grateful to Y. Fujiwara, H. Polinder, and J. Haidenbauer for providing him computational codes of their baryon-baryon interactions.
[1] S. K. Bogner, T. T. S. Kuo, and A. Schwenk, Phys. Rep. 386, 1 (2003).

[2] M. Kohno, R. Okamoto, H. Kamada, and Y. Fujiwara,, Phys. Rev. C 76, 064002 (2007).

[3] T. A. Rijken, V. G. J. Stoks, and Y. Yamamoto, Phys. Rev. C 59, 21 (1999).

[4] Y. Fujiwara, Y. Suzuki, and.C. Nakamoto, Prog. Part. Nucl. Phys. 58, 439 (2007).

[5] H. Polinder, J. Haidenbauer, and Ulf-G. Meißner, Nucl. Phys. A779, 244 (2006).

[6] H. Polinder, J. Haidenbauer, and Ulf-G. Meißner, Phys. Lett. B653, 29 (2007).

[7] Y. Fujiwara, C. Nakamoto, and Y. Suzuki, Phys. Rev. Lett. 76, 2242 (1996).
[8] H. Noumi et al., Phys. Rev. Lett. 89, 072301 (2002); 90, 049902(E) (2003).

[9] T. Harada and Y. Hirabayashi, Nucl. Phys. A759, 143 (2005).

[10] M. Kohno, Y. Fujiwara, Y. Watanabe, K. Ogata, and M. Kawai, Phys. Rev. C 74, 064613 (2006).

[11] P. K. Saha et al., Phys. Rev. C 70, 044613 (2004).

[12] M. Kohno and Y. Fujiwara, Phys. Rev. C 79, 054318 (2009).

[13] C. J. Batty, E. Friedman, and A. Gal, Phys. Lett. B335 (1994) 273; Phys. Rep. 287, 385 (1997).

[14] E. Epelbaum, W. Glöckle, and Ulf-G. Meißner, Nucl. Phys. A747, 362 (2005).

[15] K. Suzuki and S. Y. Lee, Prog. Theor. Phys. 64, 2091 
(1980).

[16] S. Y. Lee and K. Suzuki, Phys. Lett. 91B, 173 (1980).

[17] S. Okubo, Prog. Theor. Phys. 12, 603 (1954).

[18] S. Fujii, E. Epelbaum, H. Kamada, R. Okamoto, K. Suzuki, and W. Glöckle, Phys. Rev. C 70, 024003 (2004).

[19] M. Wagner, B.-J. Schaefer, J. Wambach, T. T. S. Kuo, and G. E. Brown, Phys. Rev. C 74, 054003 (2006).

[20] M. Kohno, Y. Fujiwara, T. Fujita, C. Nakamoto, and Y. Suzuki, Nucl. Rev. A674, 229 (2000).

[21] Y. Suzuki, H. Matsumura, M. Orabi, Y. Fujiwara, P. Descouvemont, M. Theeten, and D. Baye, Phys. Lett. B659, 160 (2008).

[22] K. Miyagawa, H. Kamada, W. Glöckle, and V. Stoks, Phys. Rev. C 51, 2905 (1995).

[23] A. Nogga, nucl-th/0611081.
[24] Y. Fujiwara, Y. Suzuki, M. Kohno, and K. Miyagawa, Phys. Rev. C 77, 027001 (2008).

[25] O. Hashimoto and H. Tamura, Prog. Part. Nucl. Phys. 57, 564 (2006).

[26] R. R. Scheerbaum, Nucl. Phys. A257, 77 (1976).

[27] R. S. Hayano et al., Phys. Lett. B231, 355 (1989).

[28] T. Nagae et al., Phys. Rev. Lett. 80, 1605 (1998).

[29] M. Oka, K. Shimizu, and K. Yazaki, Nucl. Phys. A464, 700 (1987).

[30] P. Khaustov et al., Phys. Rev. C61, 054603 (2000).

[31] M. Kohno and S. Hashimoto, Prog. Theor. Phys. 123 (2010), in print.

[32] T. Nagae, Nucl. Phys. A805, 486 (2008). 